\title{
ON WEYL AND BROWDER SPECTRA OF TENSOR PRODUCTS
}

\author{
C. S. KUBRUSLY \\ Catholic University of Rio de Janeiro, 22453-900, Rio de Janeiro, RJ, Brazil \\ e-mail: carlos@ele.puc-rio.br \\ and B. P. DUGGAL \\ 8 Redwood Grove, Northfields Avenue, Ealing, London W5 4SZ, England, U.K. \\ e-mail:bpduggal@yahoo.co.uk
}

(Received 12 December, 2006; revised 29 September, 2007)

\begin{abstract}
Let $A$ and $B$ be Hilbert space operators. In this paper we explore the structure of parts of the spectrum of the tensor product $A \otimes B$, and conclude some properties that follow from such a structure. We give conditions on $A$ and $B$ ensuring that $\sigma_{w}(A \otimes B)=\sigma_{w}(A) \cdot \sigma(B) \cup \sigma(A) \cdot \sigma_{w}(B)$, where $\sigma(\cdot)$ and $\sigma_{w}(\cdot)$ stand for the spectrum and Weyl spectrum, respectively. We also investigate the problem of transferring Weyl and Browder's theorems from $A$ and $B$ to their tensor product $A \otimes B$.
\end{abstract}

2000 Mathematics Subject Classification. Primary 47A80; Secondary 47A53.

1. Introduction. Let $A \otimes B$ be the tensor product of a pair of Hilbert space operators $A$ and $B$. Let $\sigma(\cdot)$ and $\sigma_{w}(\cdot)$ denote the spectrum and Weyl spectrum, respectively, and let $\sigma_{0}(\cdot)=\sigma(\cdot) \backslash \sigma_{w}(\cdot)$ be the complement of the Weyl spectrum in the spectrum.

The problem of transferring Weyl's theorem from isoloid operators $A$ and $B$ to their tensor product $A \otimes B$ was considered in this journal in [10], where the proof of the main statement (viz. if $A$ and $B$ are isoloid and satisfy Weyl's theorem, then $A \otimes B$ satisfies Weyl's theorem) stands only if it is assumed that the inclusions $\sigma_{w}(A) \cdot \sigma(B) \cup$ $\sigma(A) \cdot \sigma_{w}(B) \subseteq \sigma_{w}(A \otimes B)$ and $\sigma_{0}(A) \cdot \sigma_{0}(B) \subseteq \sigma_{0}(A \otimes B)$ hold true. Also, an example was given of a pair of nonisoloid operators $A$ and $B$ that satisfy Weyl's theorem (and also the inclusions above), but their tensor product $A \otimes B$ does not satisfy Weyl's theorem.

Although it is known that

$$
\sigma_{w}(A \otimes B) \subseteq \sigma_{w}(A) \cdot \sigma(B) \cup \sigma(A) \cdot \sigma_{w}(B)
$$

the reverse inclusion $\sigma_{w}(A) \cdot \sigma(B) \cup \sigma(A) \cdot \sigma_{w}(B) \subseteq \sigma_{w}(A \otimes B)$ remains as an open question, and so does the identity $\sigma_{w}(A \otimes B)=\sigma_{w}(A) \cdot \sigma(B) \cup \sigma(A) \cdot \sigma_{w}(B)$. If this identity holds, then we show in Proposition 5 that $\sigma_{0}(A \otimes B) \subseteq \sigma_{0}(A) \cdot \sigma_{0}(B)$. However, the reverse inclusion $\sigma_{0}(A) \cdot \sigma_{0}(B) \subseteq \sigma_{0}(A \otimes B)$ may fail even if $A$ and $B$ are isoloid operators for which $\sigma_{w}(A \otimes B)=\sigma_{w}(A) \cdot \sigma(B) \cup \sigma(A) \cdot \sigma_{w}(B)$, and both $A$ and $B$ satisfy Weyl's theorem together with their tensor product $A \otimes B$. This will be verified in Remark 2. 
The present paper is organized as follows. Notational preliminaries are posed in Section 2. The structure of parts of the spectrum of tensor products is explored in Section 3, where the spectral properties that will be required later are proved. The problem of giving conditions on $A$ and $B$ sufficient to ensure the identity

$$
\sigma_{w}(A \otimes B)=\sigma_{w}(A) \cdot \sigma(B) \cup \sigma(A) \cdot \sigma_{w}(B)
$$

is considered in Section 4. We close the paper in Section 5 by establishing conditions on $A$ and $B$ for transferring Weyl and Browder's theorems from them to their tensor product $A \otimes B$, which extend the previous results along this line.

2. Preliminaries. Let $\mathcal{H}$ be a nonzero complex Hilbert space (with inner product $\langle;\rangle$ being linear in the first argument). The single tensor product of $x \in \mathcal{H}$ and $y \in \mathcal{H}$ is a conjugate bilinear functional $x \otimes y: \mathcal{H} \times \mathcal{H} \rightarrow \mathbb{C}$ defined by

$$
(x \otimes y)(u, v)=\langle x ; u\rangle\langle y ; v\rangle \quad \text { for every } \quad(u, v) \in \mathcal{H} \times \mathcal{H} .
$$

The tensor product space $\mathcal{H} \otimes \mathcal{H}$ is the completion of the collection of all (finite) sums of single tensors, which is a Hilbert space with respect to the inner product

$$
\left\langle\sum_{i} x_{i} \otimes y_{i} ; \sum_{j} w_{j} \otimes z_{j}\right\rangle=\sum_{i} \sum_{j}\left\langle x_{i} ; w_{j}\right\rangle\left\langle y_{i} ; z_{j}\right\rangle,
$$

for every $\sum_{i} x_{i} \otimes y_{i}$ and $\sum_{j} w_{j} \otimes z_{j}$ in $\mathcal{H} \otimes \mathcal{H}$. By an operator we mean a bounded linear transformation of a normed space into itself. The tensor product of two operators $A$ and $B$ on $\mathcal{H}$ is the operator $A \otimes B$ defined by

$$
(A \otimes B) \sum_{i} x_{i} \otimes y_{i}=\sum_{i} A x_{i} \otimes B y_{i} \quad \text { for every } \quad \sum_{i} x_{i} \otimes y_{i} \in \mathcal{H} \otimes \mathcal{H},
$$

which in fact is bounded and linear on $\mathcal{H} \otimes \mathcal{H}$. For an expository paper containing the essential properties of tensor products needed here, the reader is referred to [9].

Let $T$ be an operator on a Hilbert space $\mathcal{H}$, let $\sigma(T)$ stand for the spectrum of $T$ and $\sigma_{P}(T)$ for the point spectrum (i.e., the set of all eigenvalues) of $T$, and let $\mathcal{R}(T)=T(\mathcal{H})$ and $\mathcal{N}(T)=T^{-1}\{0\}$ be the range and kernel of $T$, respectively. We recall in Proposition 0 below some well-known fundamental properties related to the spectrum of tensor products that will be required later in the sequel.

Proposition 0. Let $A$ and $B$ be operators on a Hilbert space $\mathcal{H}$. Consider the tensor product $A \otimes B$ on $\mathcal{H} \otimes \mathcal{H}$. The following assertions hold true.

(1) $\sigma(A) \cdot \sigma(B)=\sigma(A \otimes B)$.

(2) $\sigma_{P}(A) \cdot \sigma_{P}(B) \varsubsetneqq \sigma_{P}(A \otimes B)$.

Moreover, (2.a) if $0 \in \sigma_{P}(A) \cup \sigma_{P}(B)$, then $0 \in \sigma_{P}(A \otimes B)$ even if one of $\sigma_{P}(A)$ or $\sigma_{P}(B)$ is empty. Furthermore, (2.b) if $0 \neq v \in \sigma_{P}(A \otimes B)$ has an eigenvector that is a single tensor, then $v \in \sigma_{P}(A) \cdot \sigma_{P}(B)$.

(3) $\mathcal{R}(A) \otimes \mathcal{R}(B)=\mathcal{R}(A \otimes B)$.

(4) $\mathcal{N}(A) \otimes \mathcal{N}(B) \varsubsetneqq \mathcal{N}(A \otimes B)$.

The identity in (1) is a classical result from [3]. The remaining results are readily verified. It can be shown that the inclusion in (2) may be proper by using a unilateral 
shift. Indeed, if $S$ is a unilateral shift (of any multiplicity) on a Hilbert space, then $\sigma_{P}(S)$ is empty and $\sigma_{P}\left(S^{*}\right)$ is the open unit disc, and so $\sigma_{P}\left(S^{*}\right) \cdot \sigma_{P}(S)=\emptyset$. However, $\sigma_{P}\left(S^{*} \otimes S\right)=\{0\}$ (cf. [8], p. 473, 474]). Thus $\sigma_{P}\left(S^{*} \otimes S\right) \nsubseteq \sigma_{P}\left(S^{*}\right) \cdot \sigma_{P}(S)$. Moreover, the reverse inclusion in (4) fails whenever one of $A$ or $B$ is not null and the other is not injective. In fact, if $\mathcal{N}(A) \neq \mathcal{H}$ and $\mathcal{N}(B) \neq\{0\}$, then we take $x \notin \mathcal{N}(A)$ and $0 \neq y \in \mathcal{N}(B)$ so that $x \otimes y \notin \mathcal{N}(A) \otimes \mathcal{N}(B)$ but $x \otimes y \in \mathcal{N}(A \otimes B)$, since $(A \otimes B)(x \otimes y)=A x \otimes B y=A x \otimes 0=0$.

3. Some spectral properties of tensor products. We discuss some basic spectral properties of tensor products that will be needed next. For any operator $T$ let $\sigma_{P F}(T)$ be the set of all eigenvalues of finite multiplicity:

$$
\begin{aligned}
\sigma_{P F}(T) & =\left\{\lambda \in \sigma_{P}(T): \operatorname{dim} \mathcal{N}(\lambda I-T)<\infty\right\} \\
& =\{\lambda \in \mathbb{C}: 0<\operatorname{dim} \mathcal{N}(\lambda I-T)<\infty\} .
\end{aligned}
$$

Proposition 1. If $A, B$ are operators on an infinite-dimensional Hilbert space, then

$$
0 \notin \sigma_{P F}(A \otimes B) .
$$

Proof. Property (4) of Proposition 0 is a particular case of

(5) $(\mathcal{N}(A) \otimes \mathcal{H}) \cup(\mathcal{H} \otimes \mathcal{N}(B)) \varsubsetneqq \mathcal{N}(A \otimes B)$.

Indeed, if $z=\sum_{i} x_{i} \otimes y_{i} \in \mathcal{N}(A) \otimes \mathcal{H}$, then each $x_{i}$ lies in $\mathcal{N}(A)$ so that $(A \otimes B) z=(A \otimes B) \sum_{i} x_{i} \otimes y_{i}=\sum_{i} A x_{i} \otimes B y_{i}=0$, and hence $z \in \mathcal{N}(A \otimes B)$. Therefore, $(\mathcal{N}(A) \otimes \mathcal{H}) \subseteq \mathcal{N}(A \otimes B)$. Similarly, $(\mathcal{H} \otimes \mathcal{N}(B)) \subseteq \mathcal{N}(A \otimes B)$. The reverse inclusion fails if both $A$ and $B$ are not null and not infective. In fact, if $A \neq O$ and $B \neq O$, then we take $u \in \mathcal{N}(A), x \notin \mathcal{N}(A), v \in \mathcal{N}(B)$ and $y \notin \mathcal{N}(B)$ so that

$$
(A \otimes B)(u \otimes y+x \otimes v)=A u \otimes B y+A x \otimes B v=0,
$$

and hence $u \otimes y+x \otimes v \in \mathcal{N}(A \otimes B)$. However,

$$
u \otimes y+x \otimes v \notin(\mathcal{N}(A) \otimes \mathcal{H}) \cup(\mathcal{H} \otimes \mathcal{N}(B))
$$

(because $x \notin \mathcal{N}(A)$ and $y \notin \mathcal{N}(B)$ ).

Moreover, $A \otimes B$ is injective if and only if $A$ and $B$ are injective. That is,

(6) $\mathcal{N}(A)=\mathcal{N}(B)=\{0\} \quad \Longleftrightarrow \mathcal{N}(A \otimes B)=\{0\}$.

To verify the direct implication quickly suppose that $\mathcal{H}$ is separable. If $\mathcal{N}(A)=\{0\}$ and $\mathcal{N}(B)=\{0\}$, then $\mathcal{N}(A \otimes I)=\{0\}$ and $\mathcal{N}(I \otimes B)=\{0\}$ (because $I \otimes B \cong \bigoplus_{n} B$ and $A \otimes I \cong I \otimes A \cong \bigoplus_{n} A$, where $\cong$ means unitary equivalence) and so $\mathcal{N}(A \otimes B)=$ $\{0\}$, since $A \otimes B=(A \otimes I)(I \otimes B)$. (Recall that if $\mathcal{N}(S)=\{0\}$ and $\mathcal{N}(T)=\{0\}$, then $\mathcal{N}(S T)=\{0\})$. The converse follows by (5): if $\mathcal{N}(A \otimes B)=\{0\}$, then $\mathcal{N}(A)=\{0\}$ and $\mathcal{N}(B)=\{0\}$.

Furthermore, (5) also ensures that (if $\mathcal{H}$ is infinite-dimensional)

(7) $\operatorname{dim} \mathcal{N}(A) \neq 0$ or $\operatorname{dim} \mathcal{N}(B) \neq 0 \Longrightarrow \operatorname{dim} \mathcal{N}(A \otimes B)=\infty$.

In fact, since $\operatorname{dim}(\mathcal{N}(A) \otimes \mathcal{H})=\operatorname{dim} \mathcal{N}(A) \cdot \operatorname{dim} \mathcal{H}$ (cf. [11, p. 49]) and, similarly, $\operatorname{dim}(\mathcal{H} \otimes \mathcal{N}(B))=\operatorname{dim} \mathcal{H} \cdot \operatorname{dim} \mathcal{N}(B)$, it follows by (5) that $\operatorname{dim} \mathcal{N}(A \otimes B)=\infty$ whenever $\operatorname{dim} \mathcal{N}(A) \neq 0$ or $\operatorname{dim} \mathcal{N}(B) \neq 0$.

Therefore $0 \notin \sigma_{P F}(A \otimes B)$. To establish this, we reason as follows. 
If $0 \in \sigma_{P F}(A \otimes B)$, then $0 \in \sigma_{P}(A \otimes B)$, i.e., $\mathcal{N}(A \otimes B) \neq\{0\}$, and hence $\mathcal{N}(A) \neq\{0\}$ or $\mathcal{N}(B) \neq\{0\}$ according to (6). Also $\operatorname{dim} \mathcal{N}(A \otimes B)<\infty$ so that $\mathcal{N}(A)=\mathcal{N}(B)=\{0\}$ according to (7), which leads to a contradiction.

Proposition 2. Take an arbitrary pair $\{A, B\}$ of Hilbert space operators.

$$
\sigma_{P}(A) \cdot \sigma_{P}(B) \cap \sigma_{P F}(A \otimes B) \varsubsetneqq \sigma_{P F}(A) \cdot \sigma_{P F}(B) .
$$

Moreover, the identity $\sigma_{P}(A) \cdot \sigma_{P}(B) \cap \sigma_{P F}(A \otimes B)=\sigma_{P F}(A) \cdot \sigma_{P F}(B)$ holds if and only if $\sigma_{P F}(A) \cdot \sigma_{P F}(B) \subseteq \sigma_{P F}(A \otimes B)$. Furthermore,

(b) $\quad\left(\sigma_{P}(A) \backslash \sigma_{P F}(A)\right) \cdot \sigma_{P}(B) \cup \sigma_{P}(A) \cdot\left(\sigma_{P}(B) \backslash \sigma_{P F}(B)\right) \varsubsetneqq \sigma_{P}(A \otimes B) \backslash \sigma_{P F}(A \otimes B)$.

Proof. Observe that $(\lambda I-A) \otimes B+\lambda I \otimes(\mu I-B)=\lambda \mu I-A \otimes B$ for every pair of scalars $\{\lambda, \mu\}$. If $z=\sum_{i} x_{i} \otimes y_{i}$ lies in $\mathcal{N}(\lambda I-A) \otimes \mathcal{N}(\mu I-B)$, then each $x_{i}$ lies $\mathcal{N}(\lambda I-A)$ and each $y_{i}$ lies in $\mathcal{N}(\mu I-B)$, and therefore $(\lambda \mu I-A \otimes B) z=$ $[(\lambda I-A) \otimes B] z+\lambda I \otimes(\mu I-B)] z=\sum_{i}(\lambda I-A) x_{i} \otimes B y_{i}+\sum_{i} \lambda x_{i} \otimes(\mu I-B) y_{i}=0$.

Hence,

$$
\text { (8) } \mathcal{N}(\lambda I-A) \otimes \mathcal{N}(\mu I-B) \subseteq \mathcal{N}(\lambda \mu I-A \otimes B),
$$

which extends the property (4) of Proposition 0. Since the dimension of the tensor product of two Hilbert spaces is the product of their respective dimensions [11, p. 49], it follows by the inclusion in (8) that if $0<\operatorname{dim} \mathcal{N}(\lambda I-A), 0<\operatorname{dim} \mathcal{N}(\mu I-B)$, and $\operatorname{dim} \mathcal{N}(\lambda \mu I-A \otimes B)<\infty$, then we may infer that $0<\operatorname{dim} \mathcal{N}(\lambda I-A)<\infty$, $0<\operatorname{dim} \mathcal{N}(\mu I-B)<\infty$, and $0<\operatorname{dim} \mathcal{N}(\lambda \mu I-A \otimes B)$. Therefore, if $\lambda \in \sigma_{P}(A)$, $\mu \in \sigma_{P}(B)$ (so that $\lambda \mu \in \sigma_{P}(A \otimes B)$ by (2) in Proposition 0), and $\lambda \mu \in \sigma_{P F}(A \otimes B)$, then $\lambda \in \sigma_{P F}(A)$ and $\mu \in \sigma_{P F}(B)$, which proves the inclusion in (a): namely

$$
\sigma_{P}(A) \cdot \sigma_{P}(B) \cap \sigma_{P F}(A \otimes B) \subseteq \sigma_{P F}(A) \cdot \sigma_{P F}(B) .
$$

Since $\sigma_{P F}(A) \cdot \sigma_{P F}(B) \subseteq \sigma_{P}(A) \cdot \sigma_{P}(B)$ holds trivially, we may infer that, if the inclusion $\sigma_{P F}(A) \cdot \sigma_{P F}(B) \subseteq \sigma_{P F}(A \otimes B)$ holds, then we get the identity

$$
\sigma_{P}(A) \cdot \sigma_{P}(B) \cap \sigma_{P F}(A \otimes B)=\sigma_{P F}(A) \cdot \sigma_{P F}(B) ;
$$

and the converse is clear: the identity above implies $\sigma_{P F}(A) \cdot \sigma_{P F}(B) \subseteq \sigma_{P F}(A \otimes B)$. Observe that the inclusion in (b),

$$
\left(\sigma_{P}(A) \backslash \sigma_{P F}(A)\right) \cdot \sigma_{P}(B) \cup \sigma_{P}(A) \cdot\left(\sigma_{P}(B) \backslash \sigma_{P F}(B)\right) \subseteq \sigma_{P}(A \otimes B) \backslash \sigma_{P F}(A \otimes B),
$$

also follows from property (8), since the dimension of the tensor product of two Hilbert spaces is the product of their respective dimensions. To verify that the inclusions in the proposition statement are all proper, just let one of $A$ or $B$ (or both) have zero as an eigenvalue of finite multiplicity, and apply Proposition 1.

For every operator $T$ let $\sigma_{\text {iso }}(T)$ denote the set of all isolated points of $\sigma(T)$ and put $\sigma_{\text {acc }}(T)=\sigma(T) \backslash \sigma_{\text {iso }}(T)$, the set of all accumulation points of $\sigma(T)$.

Proposition 3. Let $A$ and $B$ be Hilbert spaces operators.

(a) If $\sigma_{\text {iso }}(A) \neq \varnothing$ and $\sigma_{\text {iso }}(B) \neq \varnothing$, then

$$
\sigma_{\text {iso }}(A \otimes B) \varsubsetneqq \sigma_{\text {iso }}(A) \cdot \sigma_{\text {iso }}(B) .
$$


(b) If either $\sigma_{\text {iso }}(A)=\varnothing$ or $\sigma_{\text {iso }}(B)=\varnothing$, then

$$
\sigma_{\text {iso }}(A \otimes B) \varsubsetneqq\{0\},
$$

and $\left(\mathrm{b}_{1}\right) 0 \in \sigma_{\text {iso }}(A) \cup \sigma_{\text {iso }}(B)$ whenever $\sigma_{\text {iso }}(A \otimes B)=\{0\}$.

(c) If $\sigma_{\text {iso }}(A)=\sigma_{\text {iso }}(B)=\varnothing$, then

$$
\sigma_{\text {iso }}(A \otimes B)=\varnothing
$$

Proof. First consider assertions (a) and (b). If $\sigma_{\text {iso }}(A \otimes B)=\varnothing$, then the inclusions in (a) and (b) hold trivially. Thus suppose that $\sigma_{\text {iso }}(A \otimes B) \neq \varnothing$. If one of $A$ or $B$ is quasinilpotent, then the results in (a) and (b) also hold trivially (the inclusions become the identity $\left.\sigma_{\text {iso }}(A \otimes B)=\{0\}\right)$. Thus suppose that $\sigma(A) \neq\{0\}$ and $\sigma(B) \neq\{0\}$. If $v \in \sigma_{\text {iso }}(A \otimes B)$, then $v=\lambda \mu$ is an isolated point of $\sigma(A \otimes B)=\sigma(A) . \sigma(B)$ with $\lambda \in \sigma(A)$ and $\mu \in \sigma(B)$, and so the inclusion in (a) is equivalently stated as follows.

$$
v \in \sigma_{\text {iso }}(A \otimes B) \quad \Longrightarrow \quad v=\lambda \mu \text { for some } \lambda \in \sigma_{\text {iso }}(A) \text { and } \mu \in \sigma_{\text {iso }}(B) \text {. }
$$

We shall split the proof of (a) into three parts, viz. $\left(\mathrm{a}_{1}\right),\left(\mathrm{a}_{2}\right)$ and $\left(\mathrm{a}_{3}\right)$. Then assume that $\sigma_{\text {iso }}(A) \neq \varnothing$ and $\sigma_{\text {iso }}(B) \neq \varnothing$, and take an arbitrary $\nu=\lambda \mu \in \sigma_{\text {iso }}(A \otimes B)$.

$\left(\mathrm{a}_{1}\right)$ Suppose that $\lambda \mu \neq 0$ so that $\lambda \neq 0$ and $\mu \neq 0$. If one of them, say $\lambda \in \sigma(A)$, is not an isolated point of $\sigma(A)$, then $0 \neq \lambda \in \sigma_{\text {acc }}(A)$. Thus there exists a sequence $\left\{\lambda_{n}\right\}$ of distinct points in $\sigma(A) \backslash\{\lambda\}$ such that $\lambda_{n} \rightarrow \lambda$. Therefore, since $\mu \neq 0,\left\{\lambda_{n} \mu\right\}$ is a sequence of distinct points in $\sigma(A) \cdot \sigma(B) \backslash\{\lambda \mu\}$ such that $\lambda_{n} \mu \rightarrow \lambda \mu$, and so $\lambda \mu$ is an accumulation point of $\sigma(A) \cdot \sigma(B)$, which is a contradiction.

$\left(\mathrm{a}_{2}\right)$ Now suppose that $\lambda \mu=0$ so that one of $\lambda$ or $\mu$ is zero, say $\lambda=0$. If $0=$ $\lambda \in \sigma_{\text {acc }}(A)$, then there exists a sequence $\left\{\lambda_{n}\right\}$ of distinct nonzero points in $\sigma(A) \backslash\{0\}$ such that $\lambda_{n} \rightarrow \lambda=0$. If $\mu \neq 0$, then $\left\{\lambda_{n} \mu\right\}$ is a sequence of distinct nonzero points in $\sigma(A) \cdot \sigma(B) \backslash\{0\}$ such that $\lambda_{n} \mu \rightarrow \lambda \mu=0$, and so $\lambda \mu=0$ is an accumulation point of $\sigma(A) \cdot \sigma(B)$, which is again a contradiction. If $\mu$ is also zero, then (as $\sigma(B) \neq\{0\})$ take any nonzero $\mu^{\prime} \in \sigma(B)$ so that $\lambda \mu=\lambda \mu^{\prime}=0$ with $\lambda=0$ and $\mu^{\prime} \neq 0$, thus reducing to the above case.

Therefore, in both cases (either $\lambda \mu \neq 0$ or $\lambda \mu=0$ ), if $v \in \sigma_{\text {iso }}(A \otimes B)$, then $v=\lambda \mu$ for some $\lambda \in \sigma_{\text {iso }}(A)$ and some $\mu \in \sigma_{\text {iso }}(B)$. This proves the inclusion in (a).

$\left(\mathrm{a}_{3}\right)$ Moreover, such inclusion may be proper (even if $\left.\sigma_{\text {iso }}(A \otimes B) \neq \varnothing\right)$ : take $\sigma(A)=\{1\} \cup[2,3] \cup\{4\}$ and $\sigma(B)=\{1\} \cup\{2\}$ so that

$$
\sigma(A) \cdot \sigma(B)=\{1\} \cup[2,3] \cup\{4\} \cup\{2\} \cup[4,6] \cup\{8\}=\{1\} \cup[2,3] \cup[4,6] \cup\{8\},
$$

and hence $\{1,2,4,8\}=\sigma_{\text {iso }}(A) \cdot \sigma_{\text {iso }}(B) \nsubseteq \sigma_{\text {iso }}(A \otimes B)=\{1,8\}$.

(b) Suppose that one of the operators, say $A$, is such that $\sigma_{\text {iso }}(A)=\varnothing$. If there exists $0 \neq v=\lambda \mu \in \sigma_{\text {iso }}(A \otimes B)$, then $0 \neq \lambda \in \sigma_{\text {acc }}(A)$ and $0 \neq \mu \in \sigma(B)$ so that, following the same argument as in $\left(\mathrm{a}_{1}\right), v=\lambda \mu$ is an accumulation point of $\sigma(A) \cdot \sigma(B)$, which is a contradiction. Thus $\sigma_{\text {iso }}(A \otimes B) \subseteq\{0\}$. This proves the inclusion in (b), which may be a proper one as well. For instance, if $\sigma_{\text {iso }}(A)=\varnothing, 0 \in \sigma_{\text {acc }}(A)$ and $\sigma(B)=\sigma_{\text {iso }}(B)=\{0,1\}$, then $\sigma(A) \cdot \sigma(B)=\sigma_{\text {acc }}(A)$ so that $\sigma_{\text {iso }}(A \otimes B)=\varnothing$ (i.e., $\left.\sigma(A \otimes B)=\sigma_{\text {acc }}(A)=\sigma_{\text {acc }}(A \otimes B)\right)$.

$\left(\mathrm{b}_{1}\right)$ Moreover, if $\sigma_{\text {iso }}(A \otimes B)=\{0\}$, then $0 \in \sigma(A) \cdot \sigma(B)$ and hence 0 lies in the spectrum of one of $A$ or $B$, say, $0 \in \sigma(A)$. If $\sigma_{\text {iso }}(A)=\varnothing$, then $0 \in \sigma_{\text {acc }}(A)$ and so 
(since $\sigma(B) \neq\{0\})$ the same argument as in $\left(\mathrm{a}_{2}\right)$ leads to a contradiction, and therefore $0 \in \sigma_{\text {iso }}(A)$.

(c) If $\sigma(A)=\sigma_{\text {acc }}(A)$ and $\sigma(B)=\sigma_{\text {acc }}(B)$, then it is easily verified (applying the same argument as above) that $\sigma(A \otimes B)=\sigma_{\text {acc }}(A \otimes B)$.

REMARK 1. The hypotheses $\sigma_{\text {iso }}(A) \neq \varnothing$ and $\sigma_{\text {iso }}(B) \neq \varnothing$ in Proposition 3(a) cannot be dismissed:

$$
\sigma_{\text {iso }}(A \otimes B) \nsubseteq \sigma_{\text {iso }}(A) \cdot \sigma_{\text {iso }}(B) \quad \text { if one of } \sigma_{\text {iso }}(A) \text { or } \sigma_{\text {iso }}(B) \text { is empty. }
$$

In particular,

$$
\sigma_{\text {iso }}(A)=\varnothing \text { or (but not and) } \sigma_{\text {iso }}(B)=\varnothing \quad \Longrightarrow \quad \sigma_{\text {iso }}(A \otimes B)=\varnothing,
$$

which means that $\sigma_{\text {iso }}(A \otimes B)$ may be nonempty when just one of $\sigma_{\text {iso }}(A)$ or $\sigma_{\text {iso }}(B)$ is empty. For instance, if $A$ has no isolated point in its spectrum $\left(\sigma_{\text {iso }}(A)=\varnothing\right)$ and $B$ is quasinilpotent $\left(\sigma(B)=\sigma_{\text {iso }}(B)=\{0\}\right)$, then $\sigma(A \otimes B)=\sigma_{\text {iso }}(A \otimes B)=\{0\}$. However, $\sigma_{\text {iso }}(A) \cdot \sigma_{\text {iso }}(B)=\varnothing$. Also note that

$$
\sigma_{\text {iso }}(A) \neq \varnothing \quad \text { and } \sigma_{\text {iso }}(B) \neq \varnothing \quad \Longrightarrow \quad \sigma_{\text {iso }}(A \otimes B) \neq \varnothing .
$$

That is, $\sigma_{\text {iso }}(A \otimes B)$ may be empty when both $\sigma_{\text {iso }}(A)$ and $\sigma_{\text {iso }}(B)$ are not empty.

EXAMPLE. if $\sigma(A)=[2,3] \cup\{4\} \cup[5,6]$ and $\sigma(B)=\{1\} \cup[2,10]$, then

$$
\sigma(A) \cdot \sigma(B)=[2,3] \cup\{4\} \cup[5,6] \cup[4,30] \cup[8,40] \cup[10,60]=[2,3] \cup[4,60] .
$$

Finally, recall that

$$
\sigma_{\mathrm{acc}}(A \otimes B) \subseteq \sigma_{\mathrm{acc}}(A) \cdot \sigma(B) \cup \sigma(A) \cdot \sigma_{\mathrm{acc}}(B)
$$

(as it is well known, and readily verified once $\sigma_{\text {acc }}(A \otimes B)$ is the set of all accumulation points of the product $\sigma(A) \cdot \sigma(B)$ of compact sets), and note that the inclusion may be proper. Indeed, if $A$ is quasinilpotent, then so is $A \otimes B$ for every $B$. Thus $\sigma_{\text {acc }}(A \otimes B)=\varnothing$ for every $B$, and $\sigma(A) \cdot \sigma_{\text {acc }}(B)=\{0\}$ if $\sigma_{\text {acc }}(B) \neq \varnothing$.

An operator $T$ is isoloid if isolated points of the spectrum are eigenvalues; i.e., if

$$
\sigma_{\text {iso }}(T) \subseteq \sigma_{P}(T)
$$

COROLlary 1. If $A$ and $B$ are isoloid operators on a Hilbert space $\mathcal{H}$, then so is the tensor product $A \otimes B$ on $\mathcal{H} \otimes \mathcal{H}$.

Proof. If $\sigma_{\text {iso }}(A) \neq \varnothing$ and $\sigma_{\text {iso }}(B) \neq \varnothing$ then, by property (2) in Proposition 0 and Proposition 3(a),

$$
\sigma_{\text {iso }}(A \otimes B) \subseteq \sigma_{\text {iso }}(A) \cdot \sigma_{\text {iso }}(A) \subseteq \sigma_{P}(A) \cdot \sigma_{P}(B) \subseteq \sigma_{P}(A \otimes B)
$$

and $A \otimes B$ is isoloid. Suppose just one of $\sigma_{\text {iso }}(A)$ or $\sigma_{\text {iso }}(B)$ is empty, say $\sigma_{\text {iso }}(A)=\varnothing$, so that $\sigma_{\text {iso }}(A \otimes B) \subseteq\{0\}$, according to Proposition 3(b). If $\sigma_{\text {iso }}(A \otimes B)=\{0\}$, then $0 \in \sigma_{\text {iso }}(B)$ by Proposition $3\left(\mathrm{~b}_{1}\right)$, and so $0 \in \sigma_{P}(B)$, which implies by property (2.a) of Proposition 0 that $0 \in \sigma_{P}(A \otimes B)$, and hence $\sigma_{\text {iso }}(A \otimes B) \subseteq \sigma_{P}(A \otimes B)$, so that $A \otimes B$ is again isoloid. If $\sigma_{\text {iso }}(A \otimes B)=\varnothing$, then $A \otimes B$ is trivially isoloid, which happens when $\sigma_{\text {iso }}(A)=\sigma_{\text {iso }}(B)=\varnothing$ by Proposition 3(c). 
Corollary 1 cannot be extended by replacing point spectrum with the set of eigenvalues of finite multiplicity. That is,

$$
\sigma_{\text {iso }}(A) \subseteq \sigma_{P F}(A) \text { and } \sigma_{\text {iso }}(B) \subseteq \sigma_{P F}(B) \quad \Longrightarrow \quad \sigma_{\text {iso }}(A \otimes B) \subseteq \sigma_{P F}(A \otimes B) .
$$

For instance, take the diagonal $A=\operatorname{diag}\{0, k /(k+1)\}_{k \geq 1}$ on $\ell_{+}^{2}$. This is an isoloid operator with $\sigma_{\text {iso }}(A)=\sigma_{P}(A)=\sigma_{P F}(A)=\{0, k /(k+1)\}_{k \geq 1}$ for which 0 lies in $\sigma_{\text {iso }}(A \otimes A)$ but not in $\sigma_{P F}(A \otimes A)$ by Proposition 1 . However, when the eigenvalues of finite multiplicity are intersected with the isolated points of the spectrum we get the following useful result for isoloid operators that will play a central role in the next section.

Proposition 4. If $A$ and $B$ are isoloid operators acting on an infinite-dimensional Hilbert space, then

$$
\sigma_{\text {iso }}(A \otimes B) \cap \sigma_{P F}(A \otimes B) \subseteq\left(\sigma_{\text {iso }}(A) \cap \sigma_{P F}(A)\right) \cdot\left(\sigma_{\text {iso }}(B) \cap \sigma_{P F}(B)\right) .
$$

Proof. If $\sigma_{\text {iso }}(A) \subseteq\{0\}$ or $\sigma_{\text {iso }}(B) \subseteq\{0\}$, then $\sigma_{\text {iso }}(A \otimes B) \subseteq\{0\}$, by Proposition 3(a,b,c), and so $\sigma_{\text {iso }}(A \otimes B) \cap \sigma_{P F}(A \otimes B)=\varnothing$, by Proposition 1, (since $A$ and $B$ act on an infinite-dimensional space), and the inclusion above holds. Thus suppose that $\sigma_{\text {iso }}(A) \nsubseteq\{0\}$ and $\sigma_{\text {iso }}(B) \nsubseteq\{0\}$. By Proposition 3(a),

$$
\sigma_{\text {iso }}(A \otimes B) \cap \sigma_{P F}(A \otimes B) \subseteq \sigma_{\text {iso }}(A) \cdot \sigma_{\text {iso }}(B) \cap \sigma_{P F}(A \otimes B) .
$$

Take an arbitrary $v \in \sigma_{\text {iso }}(A \otimes B) \cap \sigma_{P F}(A \otimes B)$ so that $v=\lambda \mu$ with $\lambda \in \sigma(A)$ and $\mu \in \sigma(B)$ by property (1) of Proposition 0 . Thus, according to the above inclusion and assuming that $A$ and $B$ are isoloid,

$$
\lambda \in \sigma_{\text {iso }}(A) \subseteq \sigma_{P}(A), \quad \mu \in \sigma_{\text {iso }}(B) \subseteq \sigma_{P}(B),
$$

and $\lambda \mu \in \sigma_{P F}(A \otimes B)$ so that, by Proposition 2(a),

$$
\lambda \mu \in \sigma_{P}(A) \cdot \sigma_{P}(B) \cap \sigma_{P F}(A \otimes B) \subseteq \sigma_{P F}(A) \cdot \sigma_{P F}(B) .
$$

Hence (cf. proof of Proposition 2(a)), $\lambda \in \sigma_{P F}(A)$ and $\mu \in \sigma_{P F}(B)$. Therefore,

$$
\lambda \in \sigma_{\text {iso }}(A) \cap \sigma_{P F}(A) \quad \text { and } \quad \mu \in \sigma_{\text {iso }}(B) \cap \sigma_{P F}(B),
$$

so that $v=\lambda \mu \in\left(\sigma_{\text {iso }}(A) \cap \sigma_{P F}(A)\right) \cdot\left(\sigma_{\text {iso }}(B) \cap \sigma_{P F}(B)\right)$.

Observe that the preceding inclusion for isoloid operators clearly leads to

$$
\sigma_{\text {iso }}(A \otimes B) \cap \sigma_{P F}(A \otimes B) \subseteq \sigma_{\text {iso }}(A) \cdot \sigma_{\text {iso }}(B) \cap \sigma_{P F}(A) \cdot \sigma_{P F}(B) .
$$

Corollary 2. Let $A$ and $B$ be operators on an infinite-dimensional Hilbert space. If either

(a) $\sigma_{\text {iso }}(A) \cdot \sigma_{\text {iso }}(B) \subseteq\{0\}$ (equivalently, one of $A$ or $B$, say $A$, is such that $\sigma_{\text {iso }}(A) \subseteq$ $\{0\})$,

or

(b) $A$ and $B$ are isoloid and $\sigma_{\text {iso }}(A) \cdot \sigma_{\text {iso }}(B) \cap \sigma_{P F}(A) \cdot \sigma_{P F}(B) \subseteq\{0\}$ (in particular, $A$ and $B$ are isoloid and one of $A$ or $B$, say $A$, is such that $\left.\sigma_{P F}(A) \subseteq\{0\}\right)$, then

$$
\sigma_{\text {iso }}(A \otimes B) \cap \sigma_{P F}(A \otimes B)=\varnothing .
$$


Proof. Consider the hypothesis in (a). If $\sigma_{\text {iso }}(A) \cdot \sigma_{\text {iso }}(B) \subseteq\{0\}$, then Proposition 3 ensures that $\sigma_{\text {iso }}(A \otimes B) \subseteq\{0\}$. Thus, according to Proposition 1 (since $A$ and $B$ act on an infinite-dimensional space),

$$
\sigma_{\text {iso }}(A \otimes B) \cap \sigma_{P F}(A \otimes B)=\varnothing .
$$

Now consider the hypothesis in (b). Since $A$ and $B$ are isoloid operators acting on an infinite-dimensional space, we get

$$
\sigma_{\text {iso }}(A \otimes B) \cap \sigma_{P F}(A \otimes B) \subseteq \sigma_{\text {iso }}(A) \cdot \sigma_{\text {iso }}(B) \cap \sigma_{P F}(A) \cdot \sigma_{P F}(B) \subseteq\{0\},
$$

by Proposition 4 whenever (b) holds, and so

$$
\sigma_{\text {iso }}(A \otimes B) \cap \sigma_{P F}(A \otimes B)=\varnothing
$$

according to Proposition 1.

4. The Weyl spectrum identity for tensor products. Let $T$ be an arbitrary operator on a Hilbert space $\mathcal{H}$. Set

$$
\begin{aligned}
\sigma_{0}(T)= & \left\{\lambda \in \sigma_{P}(T): \mathcal{R}(\lambda I-T)^{-}=\mathcal{R}(\lambda I-T) \neq \mathcal{H}\right. \text { and } \\
& \left.\operatorname{dim} \mathcal{N}(\lambda I-T)=\operatorname{dim} \mathcal{N}\left(\bar{\lambda} I-T^{*}\right)<\infty\right\}, \\
\sigma_{w}(T)= & \sigma(T) \backslash \sigma_{0}(T) \quad \text { and } \quad \sigma_{b}(T)=\sigma(T) \backslash\left(\sigma_{\text {iso }}(T) \cap \sigma_{0}(T)\right),
\end{aligned}
$$

where $\sigma_{w}(T)$ is precisely the Weyl spectrum of $T$ (i.e.,

$$
\sigma_{w}(T)=\{\lambda \in \mathbb{C}:(\lambda I-T) \text { is not a Fredholm operator of index zero }\}
$$

see e.g., [5]), and $\sigma_{b}(T)$ is the Browder spectrum of $T$. Also consider the essential spectrum of $T$, defined as follows.

$$
\sigma_{e}(T)=\{\lambda \in \mathbb{C}:(\lambda I-T) \text { is not a Fredholm operator }\} .
$$

Clearly, $\quad \sigma_{e}(T) \subseteq \sigma_{w}(T) \subseteq \sigma_{b}(T) \subseteq \sigma(T), \quad \sigma_{0}(T) \subseteq \sigma_{P F}(T), \quad \sigma_{\text {acc }}(T) \subseteq \sigma_{b}(T) \quad$ and $\sigma_{b}(T)=\sigma_{w}(T) \cup \sigma_{\text {acc }}(T)$. Moreover, it is also known that $\sigma_{b}(T)=\sigma_{e}(T) \cup \sigma_{\text {acc }}(T)$, and so $\sigma_{w}(T) \backslash \sigma_{e}(T) \subseteq \sigma_{\text {acc }}(T)$ (see e.g., [6]). But, in general, $\sigma_{0}(T)$ may not consist of isolated points only or, equivalently, $\sigma_{\text {acc }}(T)$ may not be included in $\sigma_{w}(T)$. An operator $T$ is said to satisfy Weyl's theorem if

$$
\sigma_{0}(T)=\sigma_{\text {iso }}(T) \cap \sigma_{P F}(T),
$$

and it is said to satisfy Browder's theorem if

$$
\sigma_{0}(T) \subseteq \sigma_{\text {iso }}(T) \cap \sigma_{P F}(T),
$$

which means that $T$ satisfies Browder's theorem if and only if

$$
\sigma_{0}(T) \subseteq \sigma_{\text {iso }}(T) \text {, that is, } \sigma_{\text {acc }}(T) \subseteq \sigma_{w}(T) \text {, equivalently, } \sigma_{w}(T)=\sigma_{b}(T) .
$$

These are the usual terminologies, and we shall stick to them, although saying that $T$ "satisfies Weyl's (or Browder's) property", rather than "satisfies Weyl's (or Browder's) theorem", would certainly be more appropriate. It is also usual to write 
$\pi_{0}(T)=\sigma_{\text {iso }}(T) \cap \sigma_{0}(T)$ and $\pi_{00}(T)=\sigma_{\text {iso }}(T) \cap \sigma_{P F}(T)$, but we shall refrain from using additional notations. It is plain that, if $T$ satisfies Weyl's theorem, then it also satisfies Browder's theorem. Note that, if $\sigma_{w}(T)=\sigma_{\text {acc }}(T)$, then $T$ satisfies Weyl's theorem. (Reason: $\sigma_{w}(T)=\sigma_{\text {acc }}(T)$ means $\sigma_{0}(T)=\sigma_{\text {iso }}(T)$, which implies $\sigma_{0}(T)=$ $\sigma_{\text {iso }}(T) \cap \sigma_{P F}(T)$ because $\sigma_{0}(T) \subseteq \sigma_{P F}(T)$.) In fact,

$T$ satisfies Browder's theorem but not Weyl's $\Longrightarrow \sigma_{w}(T) \cap \sigma_{\text {iso }}(T) \cap \sigma_{P F}(T) \neq \varnothing$.

The following results on Browder and Weyl spectra for the tensor product of any pair $\{A, B\}$ of Hilbert space operators are well known.

(i) $\sigma_{e}(A \otimes B)=\sigma_{e}(A) \cdot \sigma(B) \cup \sigma(A) \cdot \sigma_{e}(B)$,

(ii) $\sigma_{w}(A \otimes B) \subseteq \sigma_{w}(A) \cdot \sigma(B) \cup \sigma(A) \cdot \sigma_{w}(B)$,

(iii) $\sigma_{b}(A \otimes B)=\sigma_{b}(A) \cdot \sigma(B) \cup \sigma(A) \cdot \sigma_{b}(B)$,

[7, Theorem 4.2(c,f,a)]. These will be referred to as the essential spectrum identity, the Weyl spectrum inclusion, and the Browder spectrum identity, respectively. It is not known if the inclusion is proper. In other words, it is still unknown whether the Weyl spectrum identity

$$
\sigma_{w}(A \otimes B)=\sigma_{w}(A) \cdot \sigma(B) \cup \sigma(A) \cdot \sigma_{w}(B)
$$

holds for every pair of Hilbert space operators $A$ and $B$. Since in a finite-dimensional setting the Weyl spectrum identity holds trivially (because, in this case, the Browder spectrum is empty, and so are the Weyl and the essential spectra), it follows that the next results are significant only on infinite-dimensional spaces. For instance, put $\mathcal{H}=\ell_{+}^{2}$, let $A$ and $B$ be arbitrary operators on $\ell_{+}^{2}$, and let $D$ be an injective diagonal on $\ell_{+}^{2}$. It is easy to show that $\sigma_{P F}(D \otimes B)=\sigma_{P F}(A \otimes D)=\varnothing$, so that

$$
\sigma_{w}(D \otimes B)=\sigma(D \otimes B) \quad \text { and } \quad \sigma_{w}(A \otimes D)=\sigma(A \otimes D),
$$

and this leads to the Weyl spectrum identity (cf. Corollary 3 below).

For the complement $\sigma_{0}(A \otimes B)$ of $\sigma_{w}(A \otimes B)$ we get the following basic result.

Proposition 5. For every pair $\{A, B\}$ of operators on a Hilbert space $\mathcal{H}$,

(a) $0 \notin \sigma_{0}(A \otimes B)$ whenever $\mathcal{H}$ is infinite-dimensional, and

(b) $\sigma(A \otimes B) \backslash\left(\sigma_{w}(A) \cdot \sigma(B) \cup \sigma(A) \cdot \sigma_{w}(B)\right) \subseteq \sigma_{0}(A) \cdot \sigma_{0}(B)$.

Therefore, if the Weyl spectrum identity holds for $A$ and $B$, then

(b') $\sigma_{0}(A \otimes B) \subseteq \sigma_{0}(A) \cdot \sigma_{0}(B)$.

Proof. Proposition 1 says that, if $A$ and $B$ act on an infinite-dimensional Hilbert space, then $0 \notin \sigma_{P F}(A \otimes B)$. Since $\sigma_{0}(T) \subseteq \sigma_{P F}(T)$ for every Hilbert space operator $T$, we get the result in (a). Since $\sigma(A \otimes B)=\sigma(A) \cdot \sigma(B)$, it follows that

$$
\begin{aligned}
\sigma(A \otimes B) & \backslash\left(\sigma_{0}(A) \cdot \sigma_{0}(B)\right) \\
& =\left\{v \in \sigma(A) \cdot \sigma(B): v \neq \pi \rho, \text { for all }(\pi, \rho) \in \sigma_{0}(A) \times \sigma_{0}(B)\right\} \\
& \subseteq\left\{v=\lambda \mu: \lambda \in \sigma(A) \text { and } \mu \in \sigma(B), \lambda \notin \sigma_{0}(A) \text { or } \mu \notin \sigma_{0}(B)\right\} \\
& =\left\{v=\lambda \mu: \lambda \in \sigma(A) \text { and } \mu \in \sigma(B), \lambda \in \sigma_{w}(A) \text { or } \mu \in \sigma_{w}(B)\right\} \\
& =\sigma_{w}(A) \cdot \sigma(B) \cup \sigma(A) \cdot \sigma_{w}(B),
\end{aligned}
$$

which ensures the inclusion in (b), and hence the inclusion in $\left(b^{\prime}\right)$. 
REMARK 2. The inclusions in Propositions 4 and 5 may be proper, even if the operators are isoloid, satisfy Weyl's theorem together with their tensor product, and the Weyl spectrum identity holds for them. That is, there exist isoloid operators $A$ and $B$ such that $A, B$, and $A \otimes B$ satisfy Weyl's theorem, and

$$
\sigma_{w}(A \otimes B)=\sigma_{w}(A) \cdot \sigma(B) \cup \sigma(A) \cdot \sigma_{w}(B)
$$

but

$$
\sigma_{0}(A) \cdot \sigma_{0}(B) \nsubseteq \sigma_{0}(A \otimes B)
$$

or, equivalently (since they all satisfy Weyl's theorem),

$$
\left(\sigma_{\text {iso }}(A) \cap \sigma_{P F}(A)\right) \cdot\left(\sigma_{\text {iso }}(B) \cap \sigma_{P F}(B)\right) \nsubseteq \sigma_{\text {iso }}(A \otimes B) \cap \sigma_{P F}(A \otimes B) .
$$

Indeed, take

$$
A=3 \oplus I=\left(\begin{array}{ll}
3 & \\
& I
\end{array}\right) \quad \text { and } \quad B=\operatorname{diag}\{1,2\} \oplus 6 I=\left(\begin{array}{lll}
1 & & \\
& 2 & \\
& & 6 I
\end{array}\right)
$$

on $\ell_{+}^{2}$ so that

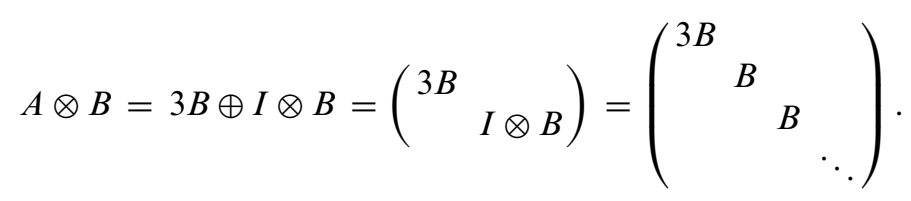

Hence

$$
\begin{aligned}
\sigma(A) & =\sigma_{\text {iso }}(A)=\sigma_{P}(A)=\{1.3\}, \quad \sigma(B)=\sigma_{\text {iso }}(B)=\sigma_{P}(B)=\{1,2,6\}, \\
\sigma_{0}(A) & =\sigma_{P F}(A)=\{3\}, \quad \sigma_{w}(A)=\{1\}, \quad \sigma_{0}(B)=\sigma_{P F}(B)=\{1,2\}, \quad \sigma_{w}(B)=\{6\}, \\
\sigma(A \otimes B) & =\sigma_{\text {iso }}(A \otimes B)=\{1,2,3,6,18\} \quad \text { and } \quad \sigma_{0}(A \otimes B)=\sigma_{P F}(A \otimes B)=\{3\} .
\end{aligned}
$$

Therefore,

$$
\{3\}=\sigma_{0}(A \otimes B) \subset \sigma_{0}(A) \cdot \sigma_{0}(B)=\{3,6\}
$$

and

$$
\begin{aligned}
\{1,2,18\} & =\sigma(A \otimes B) \backslash\left(\sigma_{0}(A) \cdot \sigma_{0}(B)\right) \\
& \subset \sigma_{w}(A) \cdot \sigma(B) \cup \sigma(A) \cdot \sigma_{w}(B)=\sigma_{w}(A \otimes B)=\{1,2,6,18\} .
\end{aligned}
$$

Since $A, B$ and $A \otimes B$ are self-adjoint, they all satisfy Weyl's theorem. Thus the inclusions in Propositions 4 and 5 may be proper even if $A$ and $B$ are isoloid operators that satisfy Weyl's theorem (with $A \otimes B$ being isoloid and also satisfying Weyl's theorem), and for which the Weyl spectrum identity holds.

By Proposition 5(a), which holds in an infinite-dimensional setting, it follows that, if $0 \in \sigma(A \otimes B)$, then $0 \in \sigma_{w}(A \otimes B)$. Recall that $\sigma_{w}(T)$ is never empty if $T$ acts on an infinite-dimensional Hilbert space, and $T$ is a commutator whenever $0 \in \sigma_{w}(T)$. Hence, 
on an infinite-dimensional space, $A \otimes B$ is a commutator whenever $0 \in \sigma(A \otimes B)$ or, equivalently, whenever $0 \in \sigma(A) \cup \sigma(B)$. See [2].

Proposition 6. Let $A$ and $B$ be operators acting on an infinite-dimensional Hilbert space and consider their tensor product $A \otimes B$. Each of the following conditions

(a) $\sigma_{w}(A \otimes B)=\sigma_{b}(A \otimes B)$,

(b) $\sigma_{e}(A) \backslash\{0\}=\sigma_{w}(A) \backslash\{0\} \quad$ and $\quad \sigma_{e}(B) \backslash\{0\}=\sigma_{w}(B) \backslash\{0\}$,

(c) $\sigma_{w}(A)=\sigma_{w}(B)=\{0\}$,

implies the Weyl spectrum identity

$$
\sigma_{w}(A \otimes B)=\sigma_{w}(A) \cdot \sigma(B) \cup \sigma(A) \cdot \sigma_{w}(B) .
$$

Proof. Since $\sigma_{w}(T) \subseteq \sigma_{b}(T)$ for every Hilbert space operator $T$, it follows from (ii) and (iii) that, if (a) holds, then

$$
\begin{aligned}
\sigma_{w}(A \otimes B) & \subseteq \sigma_{w}(A) \cdot \sigma(B) \cup \sigma(A) \cdot \sigma_{w}(B) \\
& \subseteq \sigma_{b}(A) \cdot \sigma(B) \cup \sigma(A) \cdot \sigma_{b}(B)=\sigma_{b}(A \otimes B)=\sigma_{w}(A \otimes B),
\end{aligned}
$$

and so (a) implies the Weyl spectrum identity. That is, if $A \otimes B$ satisfies Browder's theorem, then the Weyl spectrum identity holds for $A$ and $B$. Since $\sigma_{e}(T) \subseteq \sigma_{w}(T)$ for every Hilbert space operator $T$, it follows from (i) and (ii) that, if (b) holds, then

$$
\begin{aligned}
\sigma_{w}(A \otimes B) \backslash\{0\} & \subseteq \sigma_{w}(A) \backslash\{0\} \cdot \sigma(B) \backslash\{0\} \cup \sigma(A) \backslash\{0\} \cdot \sigma_{w}(B) \backslash\{0\} \\
& =\sigma_{e}(A) \backslash\{0\} \cdot \sigma(B) \backslash\{0\} \cup \sigma(A) \backslash\{0\} \cdot \sigma_{e}(B) \backslash\{0\} \\
& =\sigma_{e}(A \otimes B) \backslash\{0\} \subseteq \sigma_{w}(A \otimes B) \backslash\{0\},
\end{aligned}
$$

and so (b) implies that

$$
\sigma_{w}(A \otimes B) \backslash\{0\}=\sigma_{w}(A) \backslash\{0\} \cdot \sigma(B) \backslash\{0\} \cup \sigma(A) \backslash\{0\} \cdot \sigma_{w}(B) \backslash\{0\},
$$

which in turn implies the Weyl spectrum identity because $0 \in \sigma(A) \cup \sigma(B)$ if and only if $0 \in \sigma_{w}(A \otimes B)$, by Propositions $0(1)$ and 5(a). Recall that the Weyl spectrum is nonempty on an infinite-dimensional setting [2]. If (c) holds (i.e., if $\sigma_{w}(A)=\sigma_{w}(B)=$ $\{0\}$; e.g., if $A$ and $B$ are compact on an infinite-dimensional space [1]), then $\sigma_{w}(A \otimes B)=$ $\{0\}$ by (ii), thus satisfying the Weyl spectrum identity.

COROLlary 3. On an infinite-dimensional space, each of the following conditions

(a') $\sigma_{w}(A \otimes B)=\sigma(A \otimes B)$,

(a') $\sigma_{e}(A \otimes B)=\sigma_{b}(A \otimes B)$,

$\left(\mathrm{a}^{\prime \prime \prime}\right) \sigma_{0}(A \otimes B)=\sigma_{\text {iso }}(A \otimes B) \cap \sigma_{P F}(A \otimes B)$,

(b') $\sigma_{\text {acc }}(A) \subseteq\{0\}$ and $\sigma_{\text {acc }}(B) \subseteq\{0\}$,

implies the Weyl spectrum identity for $A$ and $B$.

Proof. Recall that $\sigma_{e}(T) \subseteq \sigma_{w}(T) \subseteq \sigma_{b}(T) \subseteq \sigma(T)$ and $\sigma_{w}(T) \backslash \sigma_{e}(T) \subseteq \sigma_{\text {acc }}(T)$ for every Hilbert space operator $T$. Thus any of $\left(\mathrm{a}^{\prime}\right)$ or $\left(\mathrm{a}^{\prime \prime}\right)$ implies the condition (a) of Proposition 6, and ( $\left.\mathrm{b}^{\prime}\right)$ (which holds, for instance, if $A$ and $B$ are compact) implies the condition (b) of Proposition 6. Since an operator that satisfies Weyl's theorem necessarily satisfies Browder's theorem, it follows that $\left(\mathrm{a}^{\prime \prime \prime}\right)$ also implies the condition (a) of Proposition 6. 
5. On Weyl's and Browder's theorems for tensor products. If $A \otimes B$ satisfies Browder's theorem, then the Weyl spectrum identity holds by Proposition 6(a). The first part of our next result ensures the converse when each of the operators satisfies Browder's theorem. The second part establishes sufficient conditions for transferring Weyl's theorem from isoloid operators to their tensor product. We assume throughout this section that all operators act on an infinite-dimensional space.

Proposition 7. Suppose that the Weyl spectrum identity holds for a pair of Hilbert space operators $A$ and $B$ :

$$
\sigma_{w}(A \otimes B)=\sigma_{w}(A) \cdot \sigma(B) \cup \sigma(A) \cdot \sigma_{w}(B) .
$$

(a) If both $A$ and B satisfy Browder's theorem, then the tensor product $A \otimes B$ satisfies Browder's theorem.

(b) If $A$ and $B$ are both isoloid and satisfy Weyl's theorem, then

$$
\sigma_{0}(A \otimes B) \subseteq \sigma_{\text {iso }}(A \otimes B) \cap \sigma_{P F}(A \otimes B) \subseteq \sigma_{0}(A) \cdot \sigma_{0}(B)
$$

Proof. (a) Recall from Remark 1 that

$$
\sigma_{\mathrm{acc}}(A \otimes B) \subseteq \sigma_{\mathrm{acc}}(A) \cdot \sigma(B) \cup \sigma(A) \cdot \sigma_{\mathrm{acc}}(B) .
$$

Thus, if $A$ and $B$ satisfy Browder's theorem, which means that $\sigma_{\text {acc }}(A) \subseteq \sigma_{w}(A)$ and $\sigma_{\text {acc }}(B) \subseteq \sigma_{w}(B)$, and if the Weyl spectrum identity holds, then

$$
\sigma_{\mathrm{acc}}(A \otimes B) \subseteq \sigma_{w}(A) \cdot \sigma(B) \cup \sigma(A) \cdot \sigma_{w}(B)=\sigma_{w}(A \otimes B),
$$

and hence $A \otimes B$ satisfies Browder's theorem, completing the proof of (a).

(b) If $A$ and $B$ satisfy Weyl's theorem, then they satisfy Browder's theorem, and so $A \otimes B$ satisfies Browder's theorem according to item (a), which means that

$$
\sigma_{0}(A \otimes B) \subseteq \sigma_{\text {iso }}(A \otimes B) \cap \sigma_{P F}(A \otimes B) .
$$

If $A$ and $B$ satisfy Weyl's theorem and are isoloid, then Proposition 4 ensures that

$$
\sigma_{\text {iso }}(A \otimes B) \cap \sigma_{P F}(A \otimes B) \subseteq \sigma_{0}(A) \cdot \sigma_{0}(B) .
$$

Thus $\sigma_{0}(A \otimes B) \subseteq \sigma_{\text {iso }}(A \otimes B) \cap \sigma_{P F}(A \otimes B) \subseteq \sigma_{0}(A) \cdot \sigma_{0}(B)$, proving (b).

Sufficient conditions for the transference of Weyl's theorem from $A$ and $B$ to $A \otimes B$ are obtained from Proposition 7(b) as in the forthcoming Corollaries 4 and 5.

Corollary 4. Suppose that the Weyl spectrum identity holds for a pair of Hilbert space operators $A$ and $B$. If then

(a) $\left(\sigma_{w}(A) \cdot \sigma(B) \cup \sigma(A) \cdot \sigma_{w}(B)\right) \cap\left(\sigma_{0}(A) \cdot \sigma_{0}(B)\right)=\varnothing$,

(b) $\left[\left(\sigma_{0}(A) \cdot \sigma_{0}(B)\right) \backslash \sigma_{0}(A \otimes B)\right] \cap \sigma_{\text {iso }}(A \otimes B) \cap \sigma_{P F}(A \otimes B)=\varnothing$.

Moreover, if $A$ and $B$ are both isoloid and satisfy Weyl's theorem, and if (b) holds, then the tensor product $A \otimes B$ satisfies Weyl's theorem.

Proof. Suppose that the Weyl spectrum identity holds for the operators $A$ and $B$. If (a) holds, then $\sigma_{0}(A) \cdot \sigma_{0}(B) \subseteq \sigma_{0}(A \otimes B)$, which implies (b). Now suppose $A$ and $B$ 
are both isoloid and satisfy Weyl's theorem. According to Proposition 7(b)

$$
\sigma_{\text {iso }}(A \otimes B) \cap \sigma_{P F}(A \otimes B) \subseteq \sigma_{0}(A) \cdot \sigma_{0}(B)
$$

so that

$$
\left(\sigma_{\text {iso }}(A \otimes B) \cap \sigma_{P F}(A \otimes B)\right) \backslash \sigma_{0}(A \otimes B) \subseteq\left(\sigma_{0}(A) \cdot \sigma_{0}(B)\right) \backslash \sigma_{0}(A \otimes B) .
$$

Thus, if (b) holds, then

$$
\left(\sigma_{\text {iso }}(A \otimes B) \cap \sigma_{P F}(A \otimes B)\right) \backslash \sigma_{0}(A \otimes B)=\varnothing,
$$

equivalently,

$$
\sigma_{\text {iso }}(A \otimes B) \cap \sigma_{P F}(A \otimes B) \subseteq \sigma_{0}(A \otimes B)
$$

Therefore, since Proposition 7(b) also ensures that

$$
\sigma_{0}(A \otimes B) \subseteq \sigma_{\text {iso }}(A \otimes B) \cap \sigma_{P F}(A \otimes B),
$$

we get

$$
\sigma_{0}(A \otimes B)=\sigma_{\text {iso }}(A \otimes B) \cap \sigma_{P F}(A \otimes B),
$$

which completes the proof.

Consequences of the preceding corollary are summarized as follows.

COROLlary 5. If the Weyl's spectrum identity holds for $A$ and $B$, both being isoloid and satisfying Weyl's theorem, and if one of the conditions (a), (b), (c) hold, where

(a) $\sigma_{0}(A) \cdot \sigma_{0}(B) \subseteq \sigma_{0}(A \otimes B)$,

(b) one of them, say $A$, is such that $\sigma_{0}(A) \subseteq\{0\}$,

(c) $\sigma_{\text {iso }}(A \otimes B) \cap \sigma_{P F}(A \otimes B) \subseteq\{0\}$,

then the tensor product $A \otimes B$ satisfies Weyl's theorem (and, in case of (b) or (c), with $\left.\sigma_{0}(A \otimes B)=\varnothing\right)$.

Proof. This follows from Corollary 4 (cf. Propositions 1 and 5).

Observe that from Proposition 5( $\left.b^{\prime}\right)$, assuming the inclusion in Corollary 5(a) or, equivalently, the intersection in Corollary 4(a), is equivalent to assuming the identity $\sigma_{0}(A \otimes B)=\sigma_{0}(A) \cdot \sigma_{0}(B)$.

Finally, Proposition 7(a) is enough to ensure that Browder's theorem transfers from $A$ and $B$ to $A \otimes B$ if and only if the Weyl spectrum identity holds.

Corollary 6. $A \otimes B$ satisfies Browder's theorem whenever both $A$ and $B$ satisfy Browder's theorem if and only if the Weyl spectrum identity holds.

Proof. Proposition 6(a) and Proposition 7(a).

For another necessary and sufficient condition for the transference of Browder's theorem from $A$ and $B$ to $A \otimes B$ see [4, Theorem 2.1]. 


\section{REFERENCES}

1. S. K. Berberian, An extension of Weyl's theorem to a class of not necessarily normal operators, Michigan Math. J. 16 (1969), 273-279.

2. S. K. Berberian, The Weyl spectrum of an operator, Indiana Univ. Math. J. 20 (1971), 529-544.

3. A. Brown and C. Pearcy, Spectra of tensor products of operators, Proc. Amer. Math. Soc. 17 (1966), 162-166. (2006)

4. B. P. Duggal, Browder-Weyl theorems, tensor products and multiplications, pre-print

5. B. P. Duggal and C. S. Kubrusly, Weyl's theorem for direct sums, Studia Sci. Math. Hungar. 44 (2007), 275-290.

6. R. Harte and W. Y. Lee, Another note on Weyl's theorem, Trans. Amer. Math. Soc. 349 (1997), 2115-2124.

7. T. Ichinose, Spectral properties of linear operators I, Trans. Amer. Math. Soc. 235 (1978), $75-113$.

8. C. S. Kubrusly, Elements of operator theory, (Birkhäuser, 2001).

9. C. S. Kubrusly, A concise introduction to tensor product, Far East J. Math. Sci. 22 (2006), 137-174.

10. Y.-M. Song and A.-H. Kim, Weyl's theorem for tensor products, Glasgow. Math. J. 46 (2004), 301-304.

11. J. Weidmann, Linear operators in Hilbert spaces, (Springer, Verlag, 1980). 